\title{
CATARACT EXTRACTION FOLLOWING VITRECTOMY AND SILICONE OIL TAMPONADE
}

\author{
R. M. BAER, W. G. AYLWARD and P. K. LEAVER \\ London
}

\section{SUMMARY}

Surgery for cataract resulting from pars plana vitrectomy combined with fluid/silicone oil exchange is often rewarding in terms of visual acuity improvement. Careful choice of surgical method and suitable modifications of standard technique reduce the incidence of serious complications. Per-operative posterior capsulotomy is a safe method of maintaining a clear visual axis after ECCE.

Pars plana vitrectomy, combined with fluid/silicone oil exchange, is a well-recognised method in the management of complex retinal detachments, ${ }^{1-5}$ enabling recovery of useful visual function in eyes that were previously deemed inoperable. The price paid for retinal reattachment, however, almost invariably includes the development of cataract in phakic eyes. ${ }^{6-8}$ Moreover, even when the silicone oil is removed at an early stage, the development of cataract, although delayed, cannot be prevented. ${ }^{9}$ The surgical management of these complex secondary cataracts is the subject of this paper.

\section{PATIENTS AND METHODS}

A series of 55 patients (56 eyes) who underwent cataract surgery was reviewed. All had previously undergone vitrectomy with silicone oil tamponade during the 5 year period from August 1987 to August 1992. All patients were under the care of a single surgeon (P.K.L.). There were 34 men and 21 women with a mean age of 56.9 years (range 19-85 years). The surgical techniques included extracapsular cataract extraction (ECCE), intracapsular cataract extraction (ICCE) and lensectomy. The majority had $\operatorname{ECCE}(n=30)$ and of these all but one eye had simultaneous insertion of a posterior chamber intraocular lens (IOL). There were 21 ICCEs and 5 lensectomies.

Correspondence to: R. M. Baer, Moorfields Eye Hospital, City Road, London EC1V 2PD, UK.

\section{SURGICAL METHODS}

Extracapsular Cataract Extraction

In eyes in which silicone oil removal was considered safe, ECCE was undertaken, either as a sequential part of a single operation or as a separate procedure, after an interval had elapsed since silicone oil removal. The method used in each event differed in two respects:

1. In eyes from which the silicone oil had previously been removed, a standard ECCE was undertaken, taking care to ensure that the lens nucleus was dislocated prior to completing the section or by hydrodissecting and hydrodelaminating the nucleus prior to expressing it. Expressing the nucleus without taking these steps can be difficult, because there is no 'support' from vitreous in the posterior segment, to aid expression.

2. In cases where the silicone oil remained in situ, it was removed, using an infusion of Hartmann's solution via the pars plana, prior to carrying out a standard ECCE. Expulsion of the nucleus in this instance can be facilitated by momentarily turning on the infusion. In both methods, the operation can be completed in the normal way, with or without implantation of a posterior chamber IOL, but in all cases it is wise to make a central opening in the posterior lens capsule as dense thickening of the capsule commonly develops and YAG laser capsulotomy can be difficult. This opening can be made quite easily with the automated cutter before inserting the IOL.

\section{Intracapsular Cataract Extraction}

In eyes in which it was deemed unsafe to remove the silicone oil, ICCE was undertaken. The presence of silicone oil can, however, make this operation less predictable than is usually the case, because the oil commonly enters the anterior chamber when the eye is opened and prevents the cryoprobe adhering to the lens capsule. The lens must then be extracted

Eye (1995) 9, 309-312 다인 1995 Royal College of Ophthalmologists 


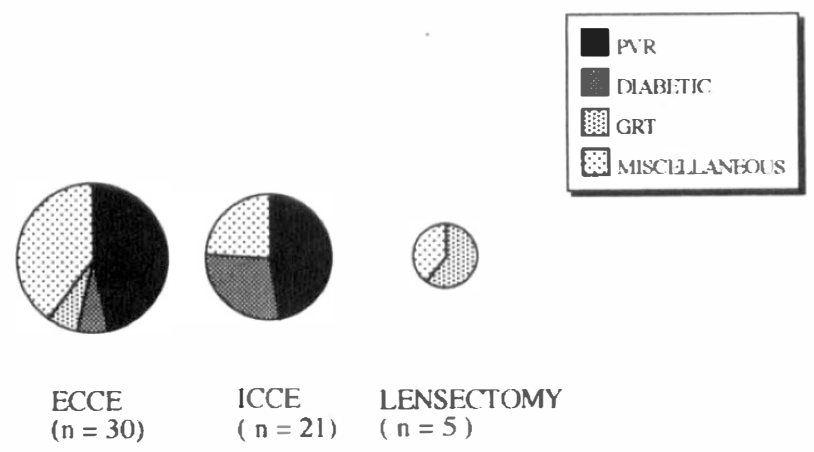

Fig. 1. Original indications for vitrectomy and silicone oil tamponade. PVR, proliferative vitreoretinopathy; GTR, giant retinal tear.

either with capsule forceps or by vectis delivery, or a combination of the two. The use of Zonulysin is helpful in these cases and the loss of some silicone oil during the procedure is usually insignificant, while, if necessary, the eye can be 'topped up' with oil at the end of the procedure, prior to re-forming the anterior chamber with Ringer's solution. A 6 o'clock peripheral iridectomy is made, using the vitrectomy cutter through the section, prior to placing the last suture (manual suction may be helpful if silicone oil obstructs the aspiration port).

\section{RESULTS}

Indications for Vitrectomy and Silicone Oil Tamponade Indications for the use of silicone oil were varied and included proliferative vitreoretinopathy (PVR; $n=24$ ), proliferative diabetic retinopathy with unrelieved traction or retinal breaks $(n=8)$, giant retinal tears (GRT; $n=5$ ) and a miscellaneous group $^{10}$ with macular holes, posterior breaks and failure of previous surgery (Fig. 1).

\section{Timing of Cataract Extraction}

The mean time between vitrectomy and cataract surgery was 13.7 months (range 6-32 months). There was some variation between the surgical groups with regard to timing of surgery. ICCE was carried out at 14.9 months (range 2-32 months) and ECCE was carried out at 12.2 months (range 1-32 months). Within the broader group of ECCE there was a difference between those having simultaneous removal of silicone oil and those who had previously had the oil removed as a separate surgical procedure, with means of 10.3 and 16.4 months respectively. In eyes where silicone oil was removed separately it had generally been done early after the original vitrectomy, prior to the development of lens opacities, mean delay being 2 months (range 1-6 months).

\section{Visual Results}

The mean length of follow-up after cataract surgery was 23 months (range 6-65 months). An overall comparison of visual acuity (VA) immediately prior to cataract removal with that at most recent followup showed an improvement of at least two lines in $46 \%$, with VA $\geqslant 6 / 60$ in $66 \%$ of cases (Fig. 2).

The degree of visual improvement varied between the three groups. After ECCE visual improvement of at least two lines was seen in $50 \%$ of cases and VA was $\geqslant 6 / 60$ in $83 \%$. (In the subset of eyes having ECCE in which a per-operative posterior capsulotomy was undertaken $(n=6)$ the VA was $\geqslant 6 / 60$ in all cases.) Following ICCE, an improvement of at least two lines was seen in $38 \%$ and VA was $\geqslant 6 / 60$ in $38 \%$. The two groups comprising ECCE and ICCE are not comparable, however, since ICCE was undertaken only in eyes that required the continuing

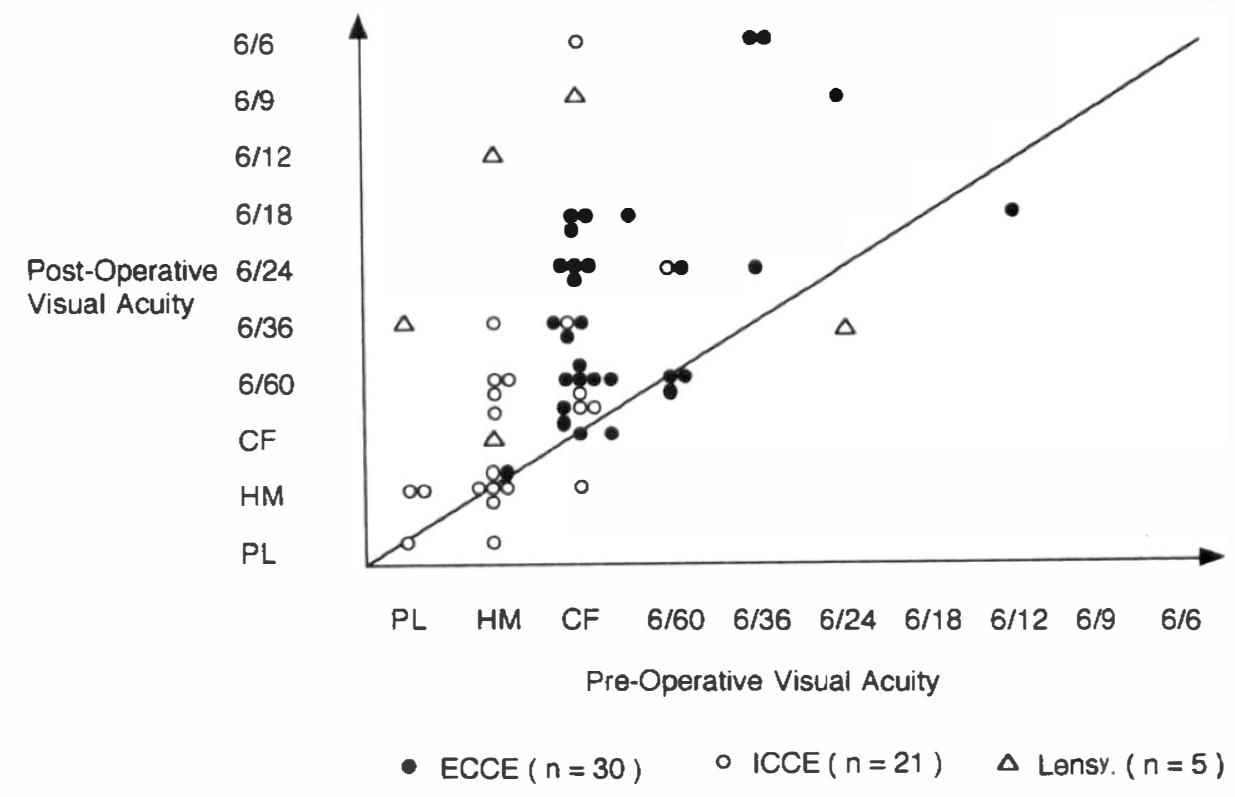

Fig. 2. Scattergram comparing the visual acuity results before and after cataract extraction. 


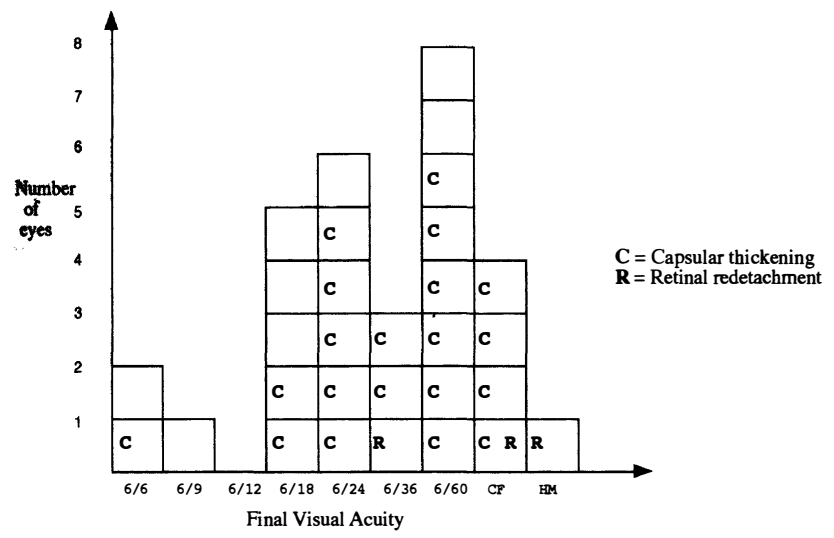

(a)

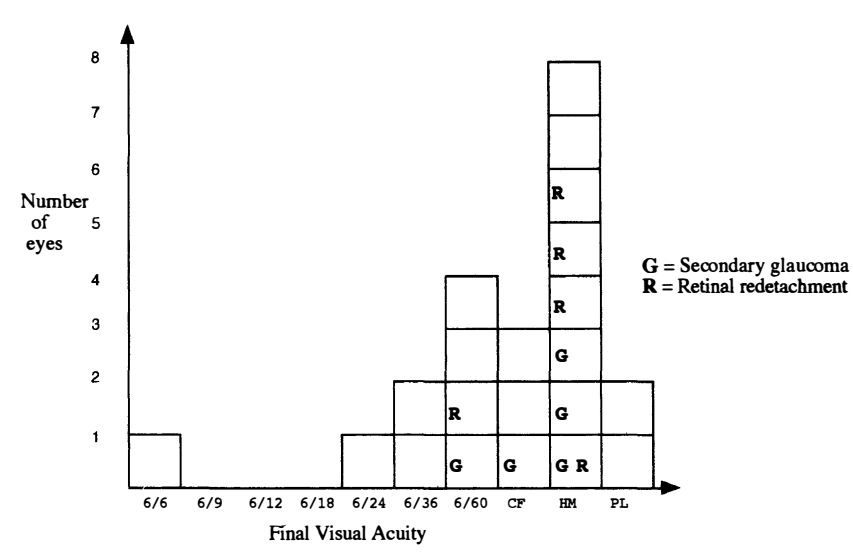

(b)

Fig. 3. Complications of ECCE (a) and ICCE (b) according to final visual acuity.

presence of silicone oil, i.e. those in which there was persistent traction or retinal breaks and/or retinal detachment. Visual results following lensectomy were the best for any single group, with $60 \%$ having visual improvement of at least two lines and $80 \%$ having final corrected VA $\geqslant 6 / 60$, best corrected acuities being achieved with contact lenses in all but one case.

\section{Per-operative Complications}

Complications during surgery varied with the surgical technique. Those encountered during ECCE included single instances of failure to implant an IOL due to splitting of the capsule and failure adequately to remove adherent cortex from the capsule. The commonest complication of ICCE was inadvertent capsular rupture requiring conversion to ECCE $(n=3)$. Capsular remnants and lens fragments were readily retrieved and removed with the vitreous cutter. On one occasion an ICCE was converted to lensectomy when oil came forward into the anterior chamber on opening the eye. In 2 of 5 eyes treated by lensectomy there were accidental sphincterotomies.

\section{Post-operative Complications}

The commonest complication of ECCE was posterior capsule opacification (Fig. 3a). After excluding eyes in which a per-operative posterior capsulotomy was performed $(n=5)$, opacification sufficient to warrant the use of YAG capsulotomy to clear the axis occurred in $80 \%$. The incidence of posterior capsule opacification of any degree was closer to $100 \%$. The extent of posterior capsular opacification was more marked and required higher power settings and number of firings to achieve opening than that usually encountered following ECCE and IOL as a primary procedure. (In one eye YAG capsulotomy was deemed impossible due to the thickness of the capsule.) Glaucoma as a de novo problem was not seen at all following ECCE and raised intraocular pressure (IOP) was recorded in only one eye, which was known to be steroid responsive prior to cataract surgery.

The commonest complication following ICCE was glaucoma (Fig. 3b). A significant rise in IOP, as a new finding, was seen in $24 \%$ of eyes. Overall, $57 \%$ of eyes had elevated IOP following ICCE, including many eyes where the diagnosis of glaucoma predated the cataract surgery.

Recurrent retinal detachment occurred in 8 eyes (overall rate $=14 \%$ ): 3 following ECCE and 5 following ICCE. All these eyes subsequently underwent at least one further retinal detachment operation with successful reattachment in three. Five of the eight eyes were deemed inoperable and no further surgery was advised (5/56).

There were no post-operative complications following lensectomy.

No cases of keratopathy have been observed in any of the groups.

\section{DISCUSSION}

The use of vitrectomy and silicone oil tamponade in the management of complex retinal detachments is becoming increasingly common. When used in phakic eyes, silicone oil almost invariably leads to cataract formation of some degree, even when it is removed at an early stage. ${ }^{9}$ The surgical approach to silicone-oil-induced cataracts is therefore of current interest.

The eyes in this series were, by definition, left phakic at the time of vitrectomy, the reason for preserving the lens at this stage being to confine the silicone oil to the posterior segment and so avoid complications associated with silicone oil in the anterior segment.

\section{Surgical Options}

The choice of surgical method is dictated by the necessity, or otherwise, for continuing silicone oil tamponade. The most effective technique in this 
series, in terms of both improvement in visual acuity and low complication rate, was shown to be ECCE, with removal of silicone oil where this had not previously been done. ECCE is only appropriate, however, where the surgeon is sufficiently confident that internal tamponade is no longer necessary to maintain retinal attachment. Eyes treated by ECCE did markedly better than those undergoing ICCE when comparing visual results in a 'reasonable' quality category $\geqslant 6 / 24$ (there were $47 \%$ of eyes achieving this level in the ECCE group compared with only $9 \%$ in the ICCE group). But although ECCE would appear to hold an advantage over ICCE, the comparison is not a valid one. Where ECCE was deemed appropriate the silicone oil had either been removed previously or was removed at the time of cataract extraction and these eyes had therefore been assessed as having secure retinas. In contrast, eyes treated by ICCE was known to have 'unsafe' retinas requiring continuing tamponade. That the results were poorer in these cases when compared with those undergoing ECCE is therefore not surprising.

ICCE is preferred in eyes containing silicone oil, because ECCE is frequently complicated by silicone oil entering the anterior chamber and causing keratopathy, while capsular thickening and subsequent YAG laser capsulotomy can often result in silicone oil entering the anterior chamber if it has not already done so.

Lensectomy was performed in 5 cases. This method was preferred in younger patients with poor visual potential, where an IOL was not deemed appropriate. In 4 of the 5 eyes retinal detachment followed trauma.

\section{Timing of Cataract Surgery}

The importance of early removal of silicone oil in limiting late complications has been stressed ${ }^{9}$ and in phakic eyes with clear lenses, this should be done at the earliest opportunity compatible with retinal security. In practice, it is unusual to remove oil earlier than 1 month following vitrectomy, but it is best undertaken within 12 weeks. Where lens opacities existed prior to vitrectomy or they appear soon after, the sense of urgency to remove the oil is diminished and it may be advantageous to wait until the cataract is more advanced, and undertake cataract extraction with oil removal as a combined procedure.

Where continuing silicone oil tamponade is deemed necessary, ICCE can be left until the cataract is sufficiently dense to seriously impair vision.

\section{Modifications of Surgical Technique}

Posterior capsular opacification following ECCE always occurs in association with intraocular silicone oil and frequently becomes so dense that YAG laser capsulotomy is difficult or impossible. ${ }^{11}$ For this reason, we recommend a per-operative surgical capsulotomy, undertaken using the vitreous cutter, before inserting the IOL.

When ICCE was carried out, a 6 o'clock peripheral iridectomy was always performed to prevent silicone oil pupil block. ${ }^{12}$

Key words: Cataract surgery, Silicone oil, Vitrectomy.

\section{REFERENCES}

1. Cibis PA, Becker B, Okun E, Canaan S. The use of liquid silicone in retinal detachment surgery. Arch Ophthalmol 1962;68:590-9.

2. Scott J. Use of liquid silicone in vitrectomised eyes. Dev Ophthalmol 1981;2:185-90.

3. Lean JS, Leaver PK, Cooling RJ, Mcleod D. Management of complex retinal detachments by vitrectomy and fluid/silicone exchange. Trans Ophthalmol Soc UK 1982;102:203-5.

4. Gonvers M. Temporary silicone oil tamponade in the management of retinal detachment with proliferative retinopathy. Am J Ophthalmol 1985;100:239-45.

5. Lucke KH, Foerster MH, Laqua H. Long-term results of vitrectomy and silicone oil in 500 cases of complicated retinal detachments. Am J Ophthalmol 1987;104:624-33.

6. Leaver PK, Grey RHB, Garner A. Silicone oil injection in the management of massive preretinal retraction. II. Late complications in 93 cases. $\mathrm{Br} \mathrm{J}$ Ophthalmol 1979;63:361-7.

7. Federman JL, Schubert HD. Complications associated with the use of silicone oil in 150 eyes after retinavitreous surgery. Ophthalmology 1988;95:870-6.

8. McCuen B, de Juan E, Landers MB, Machemer R. Silicone oil in vitreoretinal surgery. II. Results and complications. Retina 1985;5:198-205.

9. Franks WA, Leaver PK. Removal of silicone oil: rewards and penalties. Eye 1991;5:333-7.

10. Wilson-Holt N, Leaver PK. Extended criteria for vitrectomy and fluid/silicone oil exchange. Eye 1990;4:850-4.

11. Leaver PK. Complications of intraocular silicone oil. In: Ryan SJ, editor. Retina, vol 3. St Louis, MO: Mosby/Year Book, 1989:293-306.

12. Ando F. Intraocular hypertension resulting from pupillary block by silicone oil. Am J Ophthalmol 1985;99:87. 